\title{
Exploration of strong-field multiphoton double ionization, rescattering, and electron angular distribution of He atoms in intense long-wavelength laser fields: The coupled coherent-state approach
}

\author{
Jing Guo, ${ }^{1, *}$ Xue-Shen Liu, ${ }^{1, \dagger}$ and Shih-I Chu ${ }^{2, \dagger}$ \\ ${ }^{1}$ Institute of Atomic and Molecular Physics, Jilin University, Changchun 130012, People's Republic of China \\ ${ }^{2}$ Department of Chemistry, University of Kansas, Lawrence, Kansas 66045, USA
}

(Received 3 April 2010; published 3 August 2010)

\begin{abstract}
We extend the coupled coherent-state (CCS) approach to simulate the strong-field ionization of helium atoms at long wavelengths. This approach uses a basis of trajectories guided by frozen Gaussian coherent states, sampled from a Monte Carlo distribution, as the initial states of the quantum time-dependent Schrödinger equations. The CCS trajectories move over averaged potentials, which can remove the Columbic singularities exactly. The low-energy structure is predicted by our CCS calculation and a "rescattering" event is clearly identified in the higher-energy regime. In addition, the nonsequential double ionization is also explored and the rescattering event can be identified as the major mechanism. Finally, we also study the electron angular distribution of helium. It is found that the maximum angle between the electron and electric field directions becomes smaller with increase in the laser intensity and wavelength.
\end{abstract}

DOI: 10.1103/PhysRevA.82.023402

PACS number(s): $32.80 . \mathrm{Rm}, 32.60 .+\mathrm{i}$

\section{INTRODUCTION}

The study of atomic multiphoton ionization, abovethreshold ionization (ATI), nonsequential double ionization (NSDI), and high-order harmonic generation(HHG), in intense laser fields has been a subject of much interest, both theoretically and experimentally, in the last two decades [1-4]. Recently, Blaga et al. [5] have performed an experimental investigation of strong-field photoionization and observed several unexpected new features: the photoelectron energy distribution in the low-energy regime exhibits spikelike structure which becomes more prominent with mid-infrared laser wavelengths. This low-energy structure (LES) seems to be universal in all atoms and molecules but its physical origin has not yet been identified $[5,6]$. The major goal of this paper is to perform an $a b$ initio theoretical exploration of the strong-field ionization dynamics of $\mathrm{He}$ atoms in intense, long-wavelength laser fields.

A full ab initio study of two-electron atomic systems like $\mathrm{He}$ atoms in intense laser fields, involving the numerical solution of the time-dependent Schrödinger equation (TDSE) with six spatial dimensions (6D), is computationally challenging [7-9]. Accurate solution of the 6D TDSE has been recently performed for a nonperturbative study of $\mathrm{HHG}$ of $\mathrm{He}$ in intense laser pulses [10] by means of the extension of the time-dependent generalized pseudospectral method [11] to the framework of hyperspherical coordinates.

Over the last ten years, there have been many classical trajectory calculations. For example, Liu et al. [12] used a 3D semiclassical "rescattering" model to describe SFDI (shortfrequency double ionization), which considered the tunneling effects. They also used the Ammosov-Delone-Krainov method to calculate the tunneling ionization probability. Panfili et al.

\footnotetext{
*springicecat@yahoo.com.cn

†liuxs@jlu.edu.cn

†sichu@ku.edu
}

[13] also did a series of NSDI studies of atoms using the classical ensemble method, and the results are in qualitative agreement with experimental results. However, these authors did not consider the quantum effects. Xu et al. [6] also used a semiclassical method to study above-threshold ionization with a mid-infrared strong laser field, and their result is also very instructive.

In this paper, we explore and extend the coupled coherentstate (CCS) approach $[14,15]$ to the study of the LES of helium atoms as well as the associated ATI, NSDI, and rescattering phenomena. The advantages of the CCS approach are that (a) it can be extended to more degrees of freedom such as multielectron atoms and molecules; (b) the initial state can be chosen randomly; (c) certain cancellations appear in the coupling equations; and (d) the classical mechanics can be used to guide the motion of electrons. Recent applications of the CCS approach have been confined to field-free molecular dynamic studies [16-18] with one exception [19]. In this paper, the potential and advantage of the CCS approach will be further explored and applied to the study of several strong-field atomic ionization processes in longer wavelengths of current interest. In our calculation, we consider quantum effects and use the classical trajectories to guide the motion of the wave packet.

The paper is organized as follows. In Sec. II, we present the essence of the CCS method for the treatment of strongfield atomic ionization. In Sec. III, we extend the CCS approach to the study of (a) the low-energy electron structure of helium atoms, (b) mechanisms of NSDI, and (c) the photoelectron angular distributions in different laser wavelengths and intensities. This is followed by the conclusion in Sec. IV.

\section{COUPLED COHERENT-STATE THEORY}

Assuming $\mathbf{q}$ is the position and $\mathbf{p}$ is the momentum of a system at an instant of time, then the classical motion can be simply described in phase space with the variables $(\mathbf{q}, \mathbf{p})$. 
Instead, this motion can also be described in the complex plane by $\mathbf{z}$ and the complex conjugate $\mathbf{z}^{*}$, which are defined as follows:

$$
\begin{aligned}
& \mathbf{z}=\left(\frac{\gamma}{2}\right)^{1 / 2} \mathbf{q}-\frac{i}{\hbar}\left(\frac{1}{2 \gamma}\right)^{1 / 2} \mathbf{p}, \\
& \mathbf{z}^{*}=\left(\frac{\gamma}{2}\right)^{1 / 2} \mathbf{q}+\frac{i}{\hbar}\left(\frac{1}{2 \gamma}\right)^{1 / 2} \mathbf{p},
\end{aligned}
$$

where $\gamma=m \omega / \hbar$ is the width parameter, $m$ is the electron mass, $\omega$ is the interior frequency, and here we set $\omega=1$. So in this case $\gamma=1$ in atomic units. Based on both the literature and the calculation, we note that the value of $\gamma$ does not have much effect on the results.

In the coordinate representation, the coherent state (CS) $|\mathbf{z}\rangle$ in the phase space can be expressed as [14]

$$
\langle\mathbf{x} \mid \mathbf{z}\rangle=\left(\frac{\gamma}{\pi}\right)^{1 / 4} \exp \left(-\frac{\gamma}{2}(\mathbf{x}-\mathbf{q})^{2}+\frac{i}{\hbar} \mathbf{p}(\mathbf{x}-\mathbf{q})+\frac{i \mathbf{p q}}{2 \hbar}\right) \text {. }
$$

Each CS is a product of a single-particle wave packet. Each member of the CS ensemble follows its own quantum trajectory $(\mathbf{q}, \mathbf{p})$, and the quantum trajectories are determined by the Hamiltonian with quantum corrections [15]

$$
\begin{aligned}
& \frac{d \mathbf{z}}{d t}=-\frac{i}{\hbar} \frac{\partial \mathbf{H}_{\mathrm{ord}}\left(\mathbf{z}^{*}, \mathbf{z}\right)}{\partial \mathbf{z}^{*}}, \\
& \frac{d \mathbf{z}^{*}}{d t}=\frac{i}{\hbar} \frac{\partial \mathbf{H}_{\mathrm{ord}}\left(\mathbf{z}^{*}, \mathbf{z}\right)}{\partial \mathbf{z}},
\end{aligned}
$$

where $\quad \mathbf{H}_{\text {ord }}\left(\mathbf{z}^{*}, \mathbf{z}\right)=\langle\mathbf{z}|\hat{\mathbf{H}}| \mathbf{z}\rangle \quad$ represents the diagonal elements of the Hamiltonian matrix. The ordered Hamiltonian $\mathbf{H}_{\text {ord }}\left(\mathbf{z}^{*}, \mathbf{z}\right)$ is often conveniently derived by expressing $\mathbf{H}$ in terms of creation and annihilation operators, reordered such that $\mathbf{a}^{\dagger}$ is on the left and $\mathbf{a}$ is on the right,

$$
\mathbf{H}(\mathbf{p}, \mathbf{q})=\mathbf{H}\left(\mathbf{a}^{\dagger}, \mathbf{a}\right)
$$

For the helium case, the field-free Hamiltonian is

$$
\mathbf{H}=\frac{\mathbf{p}_{1}^{2}+\mathbf{p}_{2}^{2}}{2 m}-\frac{2}{\left|\mathbf{R}_{\mathbf{1}}\right|}-\frac{2}{\left|\mathbf{R}_{\mathbf{2}}\right|}+\frac{1}{\left|\mathbf{R}_{\mathbf{1 2}}\right|},
$$

where $m$ is the electron mass, $\mathbf{p}_{1}$ and $\mathbf{p}_{2}$ are the momenta of the two electrons, $\mathbf{R}_{1}$ and $\mathbf{R}_{2}$ represent the distances between individual electron and the nucleus, respectively, and $\mathbf{R}_{12}$ is the distance between the two electrons.

The coordinate $\mathbf{q}$ and momentum operator $\mathbf{p}$ are given by

$$
\begin{aligned}
& \mathbf{q}=\sqrt{\frac{\hbar}{2 m \omega}}\left(\mathbf{a}^{\dagger}+\mathbf{a}\right), \\
& \mathbf{p}=i \sqrt{\frac{\hbar m \omega}{2}}\left(\mathbf{a}^{\dagger}-\mathbf{a}\right) .
\end{aligned}
$$

The idea is to employ an ensemble of $N$ points in the six-dimensional Cartesian phase space, $(\mathbf{Q}, \mathbf{P})=$ $\left(x_{1}, y_{1}, z_{1}, x_{2}, y_{2}, z_{2}, p_{x_{1}}, p_{y_{1}}, p_{z_{1}}, p_{x_{2}}, p_{y_{2}}, p_{z_{2}}\right)$, of the two electrons as the centers of $N$ Gaussian wave packets shown in Eq. (3), which constitute a nonorthogonal time-dependent quantum basis set $\left|\mathbf{z}_{\mathrm{n}}\right\rangle$, where $n=1,2, \ldots, N$. The corresponding $6 \mathrm{D}$ coherent states are $\left|\mathbf{z}_{n}\right\rangle=\left|\mathbf{z}_{1 n}\right\rangle\left|\mathbf{z}_{2 n}\right\rangle$, each of which is a product of two single-electron 3D coherent states $\left|\mathbf{z}_{i n}\right\rangle=\left|\mathbf{z}_{\text {inx }}\right\rangle\left|\mathbf{z}_{\text {iny }}\right\rangle\left|\mathbf{z}_{\text {inz }}\right\rangle, \quad i=1,2$. For the field-free wave function part, the initial two-electron ground state is generated in the Gaussian basis shown in Eq. (3) by a coherent-state variation of the diffusion Monte Carlo procedure [19], which involves solving the time-dependent Schrödinger equation on a fixed grid, but in imaginary time.

We can also use the CCS method to remove the singularity exactly in the Coulomb potential between electron and core at the origin $\mathbf{R}_{c}$ [19],

$$
\left\langle\mathbf{z}\left|\frac{1}{\left|\mathbf{r}-\mathbf{R}_{\mathbf{c}}\right|}\right| \mathbf{z}^{\prime}\right\rangle=\left\langle\mathbf{z} \mid \mathbf{z}^{\prime}\right\rangle \frac{1}{\rho} \operatorname{erf}(\sqrt{\gamma} \rho) .
$$

where erf is the complex error function, $\rho=\frac{\mathbf{z}^{*}+\mathbf{z}^{\prime}}{\sqrt{2 \gamma}}-\mathbf{R}_{\mathrm{c}}$, and $\rho=\sqrt{\boldsymbol{\rho} \cdot \boldsymbol{\rho}}$, where $\mathbf{r}$ is the distance between the nucleus and electron. In this paper we define $\mathbf{R}_{c}=\mathbf{0}$.

Similarly, the two-electron integral can be expressed as

$\left\langle\mathbf{z}_{1}, \mathbf{z}_{2}\left|\frac{1}{\left|\mathbf{R}_{12}\right|}\right| \mathbf{z}_{1}^{\prime}, \mathbf{z}_{2}^{\prime}\right\rangle=\left\langle\mathbf{z}_{1} \mid \mathbf{z}_{1}^{\prime}\right\rangle\left\langle\mathbf{z}_{2} \mid \mathbf{z}_{2}^{\prime}\right\rangle \frac{1}{\rho_{12}} \operatorname{erf}\left(\sqrt{\gamma / 2} \rho_{12}\right)$,

where $\boldsymbol{\rho}_{1}=\frac{\mathbf{z}_{1}^{*}+\mathbf{z}_{1}^{\prime}}{\sqrt{2 \gamma}}-\mathbf{R}_{\mathrm{c}}, \quad \rho_{1}=\sqrt{\boldsymbol{\rho}_{1} \cdot \boldsymbol{\rho}_{1}}, \quad \boldsymbol{\rho}_{2}=\frac{\mathbf{z}_{2}^{*}+\mathbf{z}_{2}^{\prime}}{\sqrt{2 \gamma}}-\mathbf{R}_{\mathrm{c}}$, $\boldsymbol{\rho}_{12}=\frac{\mathbf{z}_{1}^{*}+\mathbf{z}_{1}^{\prime}}{\sqrt{2 \gamma}}-\frac{\mathbf{z}_{2}^{*}+\mathbf{z}_{2}^{\prime}}{\sqrt{2 \gamma}}$, and $\rho_{12}=\sqrt{\boldsymbol{\rho}_{12} \cdot \boldsymbol{\rho}_{12}} \cdot \boldsymbol{\rho}_{1}, \boldsymbol{\rho}_{2}$, and $\boldsymbol{\rho}_{12}$ are the coherent-state representations of the electron-nucleus and electron-electron separations, respectively. After that we can get the final form of the ordered Hamiltonian of helium in an intense laser field as follows:

$$
\begin{aligned}
\mathbf{H}_{\text {ord }}= & \frac{m \omega}{4}\left[\left(\mathbf{z}_{1}^{*}\right)^{2}-2 \mathbf{z}_{1}^{*} \mathbf{z}_{1}+\mathbf{z}_{1}^{2}+\left(\mathbf{z}_{2}^{*}\right)^{2}-2 \mathbf{z}_{2}^{*} \mathbf{z}_{2}+\mathbf{z}_{2}^{2}-2\right] \\
& +E(t)\left(\rho_{1}+\boldsymbol{\rho}_{2}\right)-\left(\frac{2}{\left|\rho_{1}\right|} \operatorname{erf}\left(\left|\rho_{1}\right|\right)+\frac{2}{\left|\rho_{2}\right|} \operatorname{erf}\left(\left|\boldsymbol{\rho}_{2}\right|\right)\right) \\
& +\frac{1}{\left|\rho_{12}\right|} \operatorname{erf}\left(\frac{\left|\rho_{12}\right|}{\sqrt{2}}\right),
\end{aligned}
$$

where $E(t)$ represents the external laser field. The detailed numerical procedure can be divided into several steps [17].

First, we calculated $\mathbf{z}_{i n}(t), i=1,2, n=1, \ldots, N$, by a fourth-order symplectic formula [20] when a group of initial values of $\mathbf{z}_{i n}(0)$ is given. After we obtain $\mathbf{z}_{i n}(t)$, we can get the corresponding coordinate and momentum distributions as a function of time, (Q,P), by Eqs. (1) and (2).

For each time step, we should calculate the wave function $\Phi(\mathbf{z}, t)$ in the coherent-state representation. The time-dependent Schrödinger equation in the coherentstate representation [14] is

$$
\begin{aligned}
\frac{d \Phi(z, t)}{d t}= & \frac{i}{\hbar} \frac{d S(\mathbf{z}, t)}{d t} \Phi(\mathbf{z}, t) \\
& -\frac{i}{\hbar} \int\left\langle\mathbf{z} \mid \mathbf{z}^{\prime}\right\rangle \delta^{2} \mathbf{H}_{\mathrm{ord}}\left(\mathbf{z}^{*}, \mathbf{z}^{\prime}\right) \psi\left(\mathbf{z}^{\prime}, t\right) \frac{d^{2} \mathbf{z}^{\prime}}{\pi},
\end{aligned}
$$

where the overlap integral is $\left\langle\mathbf{z} \mid \mathbf{z}^{\prime}\right\rangle=\exp \left[\mathbf{z}^{*} \mathbf{z}^{\prime}-\right.$ $\left.\left(\left|\mathbf{z}^{\prime}\right|^{2} / 2\right)-\left(|\mathbf{z}|^{2} / 2\right)\right]$, and, from the above equations using $\mathbf{z}_{i n}(t), i=1,2, n=1, \ldots, N$, the action $S\left(z_{n}, t\right)$ is 
given by

$$
\begin{aligned}
S\left(z_{n}, t\right)= & \int\left[\frac { i \hbar } { 2 } \left(z_{1 n}^{*} \frac{d z_{1 n}}{d t}+z_{2 n}^{*} \frac{d z_{2 n}}{d t}-\frac{d z_{1 n}^{*}}{d t} z_{1 n}\right.\right. \\
& \left.\left.-\frac{d z_{2 n}^{*}}{d t} z_{2 n}\right)-\mathbf{H}_{\text {ord }}\right] d t .
\end{aligned}
$$

The second order of the ordered Hamiltonian $\mathbf{H}_{\text {ord }}$ can be calculated as

$$
\delta^{2} \mathbf{H}_{\text {ord }}\left(\mathbf{z}^{*}, \mathbf{z}^{\prime}\right)=\frac{1}{2} \frac{\partial^{2} \mathbf{H}_{\text {ord }}\left(\mathbf{z}^{*}, \mathbf{z}\right)}{\partial \mathbf{z}^{2}}\left(\mathbf{z}^{\prime}-\mathbf{z}\right)^{2} .
$$

In order to solve this equation, we can write the wave function as $\Phi(\mathbf{z}, t)=C\left(\mathbf{z}_{n}, t\right) \exp \left[\frac{i}{\hbar} S\left(\mathbf{z}_{n}, t\right)\right], n=1, \ldots, N$. We can obtain the equations for the factor $C\left(\mathbf{z}_{n}, t\right)$ first; then we calculate the integral on the grid points $\mathbf{z}_{i n}(t)$ for $i=1,2, n=$ $1, \ldots, N[14]$ :

$$
\begin{aligned}
\frac{d C\left(z_{n}, t\right)}{d t}= & -\frac{i}{\hbar} \int\left\langle\mathbf{z}_{n} \mid \mathbf{z}_{n}{ }^{\prime}\right\rangle \delta^{2} \mathbf{H}_{\mathrm{ord}}\left(\mathbf{z}_{n}^{*}, \mathbf{z}_{n}{ }^{\prime}\right) \\
& \times \exp \left(\frac{i}{\hbar}\left[S\left(\mathbf{z}_{n}{ }^{\prime}, t\right)-S\left(\mathbf{z}_{n}, t\right)\right] C\left(\mathbf{z}_{n}{ }^{\prime}, t\right)\right) \frac{d^{2} \mathbf{z}_{n}{ }^{\prime}}{\pi} .
\end{aligned}
$$

After solving the above equations numerically, we can finally get the total wave function of the system as a function of time in the phase space $\Phi(\mathbf{z}, t)$. Then we can do a transformation to get the wave function in the coordinate representation, $\psi(\mathbf{x}, t)$. The ionization probability can now be determined as

$$
I_{p}=1-|\psi(\mathbf{x}, t)|^{2} .
$$

The double-ionization yield can now be calculated as the probability for both electrons to be separated from the nucleus by more than 12 a.u. after exposure to the laser pulse. After calculating the total ionization probability $I_{p}$, we can determine the electron angular distribution $P_{\theta}=\partial I_{p} / \partial \theta$.

\section{RESULTS AND DISCUSSION}

The present simulation was performed on an initial static grid of 100000 coherent states, randomly selected from a Gaussian distribution with the two electrons symmetrically distributed with respect to the nucleus, which reflects the symmetry of the initial wave function. Every grid point should lie below the classical escape energy, in the absence of the laser field. This ensures that no CS can escape from the Coulombic well before the field is switched on.

\section{A. The low-energy structure of helium}

Figure 1 shows the LES spectra of helium calculated at a long wavelength of $3000 \mathrm{~nm}$. The electron yield increases rapidly first, then decreases after reaching a sharp peak. The LES structure can be characterized by a peak energy (about $1.9 \mathrm{eV}$ ) and high-energy plateau (around $10.1 \mathrm{eV}$ ) defined by the break in the slope. We also calculate the LES region without the Coulomb interaction, and find that the figure is totally different from Fig. 1. Using the semiclassical method, $\mathrm{Xu}$ et al. [6] also presented the LES and they inferred that the LES peak is due to the Coulomb interaction. From our results, we

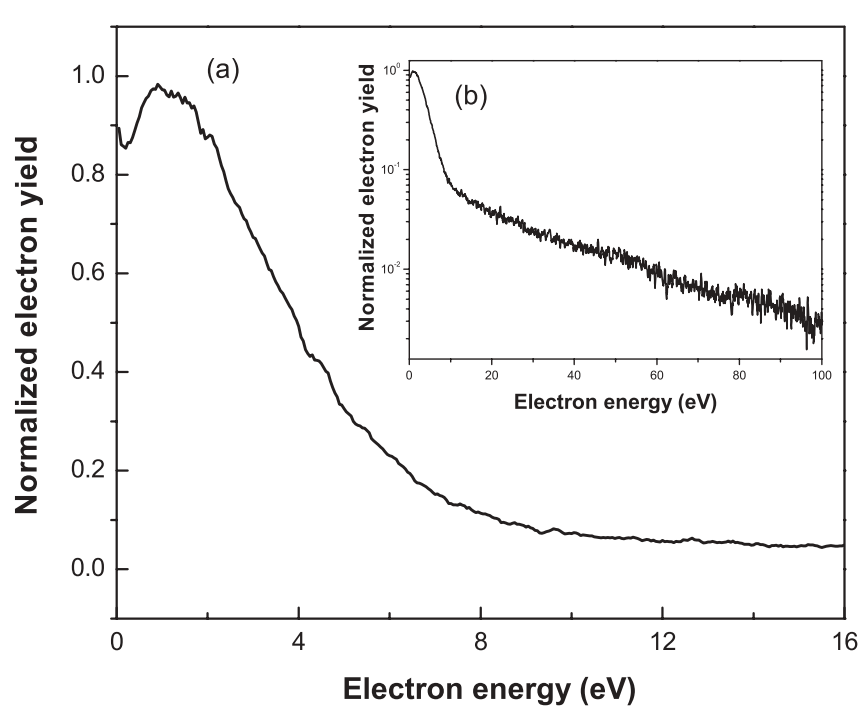

FIG. 1. Low-energy region of the photoelectron spectra for helium produced by $100 \mathrm{TW} \mathrm{cm}^{-2}, 3000 \mathrm{~nm}$ laser pulses, (a) is the partially enlarged picture of (b).

also believe the LES peak can be attributed to the Coulombic interaction. Furthermore, the LES width is much greater than the photon energy, and its integrated yield can contain up to half of the electron emission. This is for the linearly polarized laser case. The slope of electron yield decreases rapidly until a saturation point is reached (around $10 \mathrm{eV}$ ); it then decreases more slowly because of rescattering. We can see the behavior of the directly ionized and rescattered electrons in the tails of the LES spectra. Our numerical solutions of the CCS equations successfully reproduce the characteristic LES region, showing the existence of the LES as a universal feature in atoms.

Next we explore the LES dependence of the laser characteristics. Figure 2 shows the tendency of the LES behavior in helium at $2000 \mathrm{~nm}$ as a function of laser intensity. As the intensity rises at constant wavelength, the normalized LES region becomes wider with increase of the laser intensity.

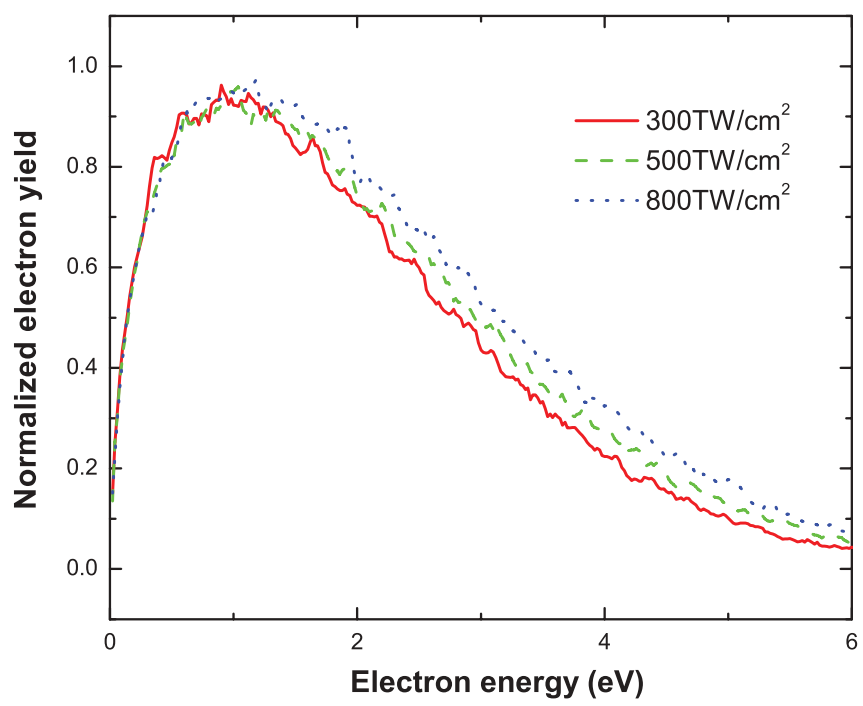

FIG. 2. (Color online) Progression of the LES in helium at $2000 \mathrm{~nm}$ as a function of laser intensity. 


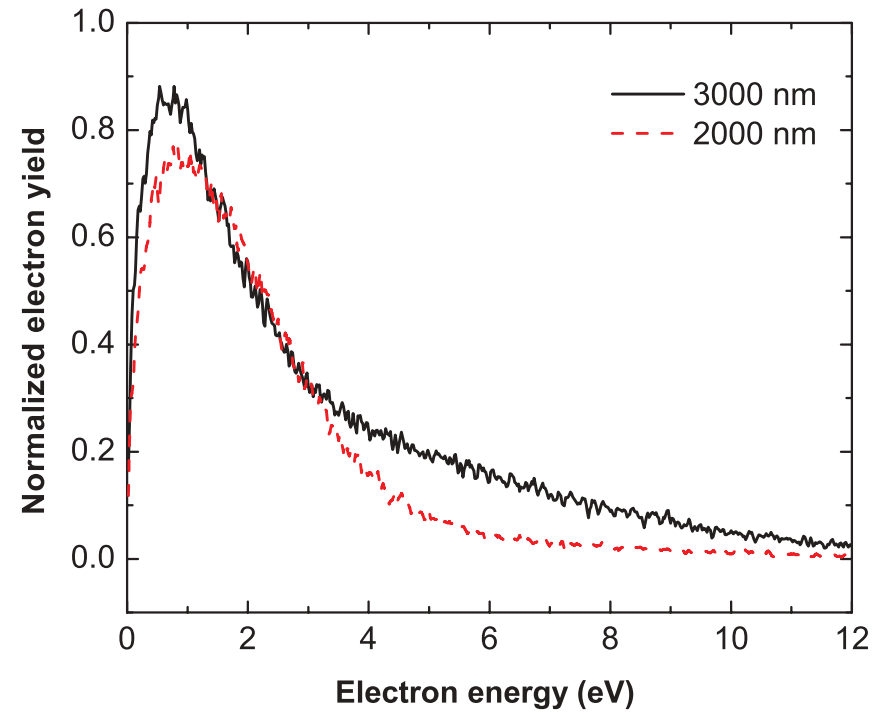

FIG. 3. (Color online) Behavior of the LES pattern of helium as a function of wavelength for laser intensity at $100 \mathrm{TW} / \mathrm{cm}^{2}$.

Figure 3 shows the LES of helium as a function of wavelength. We see that, as the wavelength increases, the LES feature becomes more visible and broadens in width, similar to the experimental results for argon [5].

\section{B. Mechanisms for nonsequential double ionization}

It is speculated that the dominant mechanism of nonsequential double ionization is due to recollisions [8,21]. This is confirmed in our analysis by inspection of the trajectories of the coherent states, because the double-ionization yield is due to the CSs whose trajectories lead to escape of both electrons. Figure 4(a) shows a typical trajectory leading eventually to double ionization at a field intensity in the region of nonsequential double ionization. The departure of the first electron from the core at $T \approx 0.8$ optical cycles is followed by multiple recollisions (oscillations in $|r|$ ), leading to simultaneous and correlated escape of the two electrons at $T \approx 3.1$ optical cycles. It shows the departure of the first electron, and then the second one recollides with the first one, followed by the correlated escape. Figure 4(b) exhibits the nonsequential double-ionization mechanism by showing the energy of the two electrons. We can see that the two electrons stay in the bound states and oscillate around some equilibrium position first. After that, one of the electrons kicks out and comes back later to the core and recollides with the second electron several times, allowing both to escape eventually.

Another way to show the NSDI from a typical quantum simulation is indicated in Fig. 5 for the laser intensity $I=$ $800 \mathrm{TW} / \mathrm{cm}^{2}$. Figure 5 shows the time evolution of the position of two electrons along the $x$ axes in a half cycle. Population along the axes in Fig. 5 indicates that one electron is near the core and the other is far away. This is a signature of single-electron excitation or ionization. Population moving out into the various quadrants indicates double ionization. In the first frame, we can see that the double-ionization trajectories mostly emerged in the first quadrant. In the second frame, the number of strong double-ionization trajectories is reduced. In the third and fourth frames, we see strong double-ionization "jets" emerge from the vicinity of the origin. In the last frame, the double-ionization trajectories mostly emerged in the third quadrant. These are the trajectories of two electrons emerging together on the same side of the nucleus, which refers to a recollision event. It should be mentioned that such jets form when the laser field is near maximum strength and there is peak barrier suppression, so the highest-energy recollision events could not take place near the half cycle. By $t=3.5$ optical cycles, the appearance of the first and third quadrants would be approximately exchanged. The whole doubly ionized population visible in the plot is in the third quadrant, indicating that both electrons are on the same side of the nucleus. It is also called a "slow-down" process and has been predicted previously by Eberly et al. [13].

\section{The photoelectron angular distribution of helium with different laser intensities and wavelengths}

Figure 6 shows the angular distribution of helium with different wavelengths in intense laser fields, which looks like a dumbbell shape. The maximum angular distribution is close to $120^{\circ}$ at first, but with increasing wavelength, the angular distribution becomes closer to the core and the maximum angle is smaller. It should be mentioned that the angular distribution is nearly the same between $45^{\circ}$ and $135^{\circ}$ especially at higher intensities. Figure 7 shows the angular distribution of
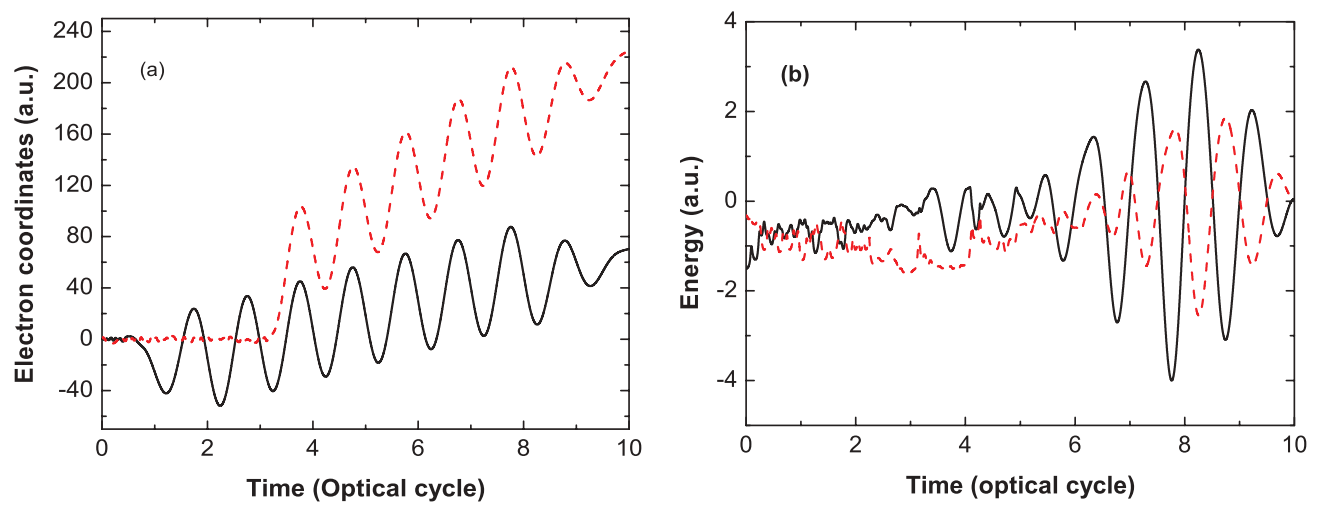

FIG. 4. (Color online) Position (a) and energy (b) distribution of a typical trajectory as a function of time for a linearly polarized laser field. The red (dashed) and black (solid) lines track the two electrons. The laser intensity is $200 \mathrm{TW} / \mathrm{cm}^{2}$. 

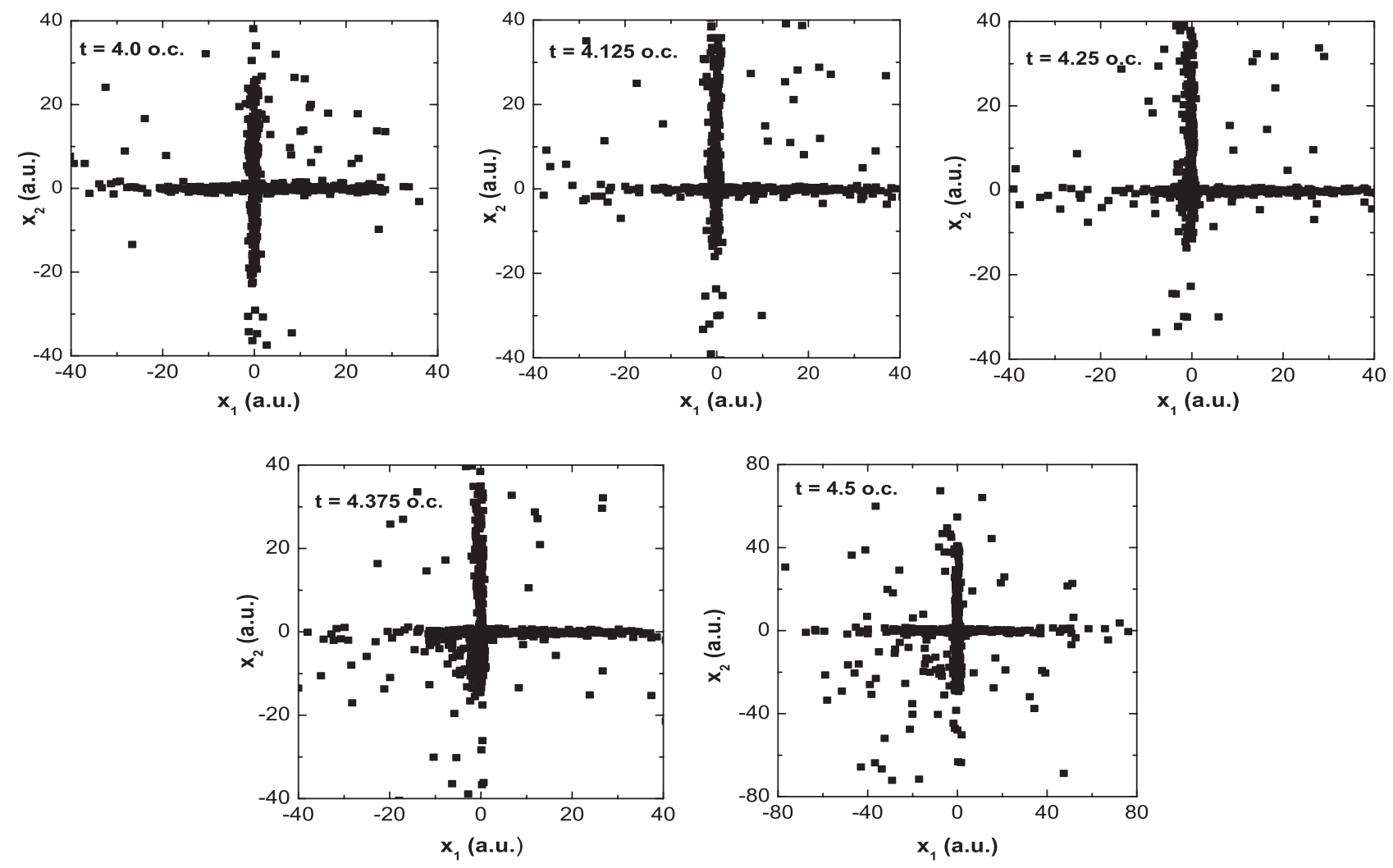

FIG. 5. Time evolution of the position of the two-electron trajectories (in the $x$ direction) which eventually participate in double ionization. The laser wavelength and intensity are $800 \mathrm{~nm}$ and $200 \mathrm{TW} / \mathrm{cm}^{2}$, respectively.

helium with different laser intensities at $2000 \mathrm{~nm}$. We can see that the angular distribution is closer to the core and the maximum angle is smaller with increasing intensity. The maximum angular distribution is close to $135^{\circ}$ at first. However, with increasing intensity, the angular distribution becomes much closer to the core, and the maximum angle becomes smaller. Also the angular distribution is similar between $30^{\circ}$ and $150^{\circ}$ especially in the higher-intensity regime. This behavior can be explained as follows: According to tunneling theory [22],

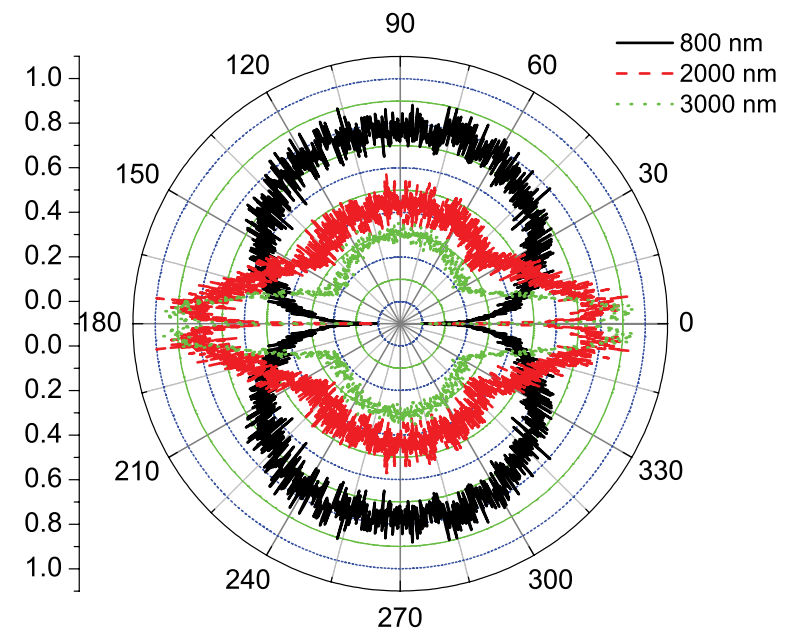

FIG. 6. (Color online) Angular distribution of helium with different wavelengths in intense laser fields; the intensity is $800 \mathrm{TW} / \mathrm{cm}^{2}$. over the entire range, the logarithmic plot suggests that the LES obeys a simple scaling law which implies that $\sin \theta$ is proportional to $E^{-1}$ and $\gamma^{-1}$. So the angle between the electron and electric field directions becomes smaller with increasing intensity and wavelength as well. It can also be explained as follows: As the wavelength or intensity increases, the ionization dynamics changes from multiphoton ionization to tunneling ionization, and it is the basic feature of tunneling ionization that the electrons tend to move along the laser field direction.

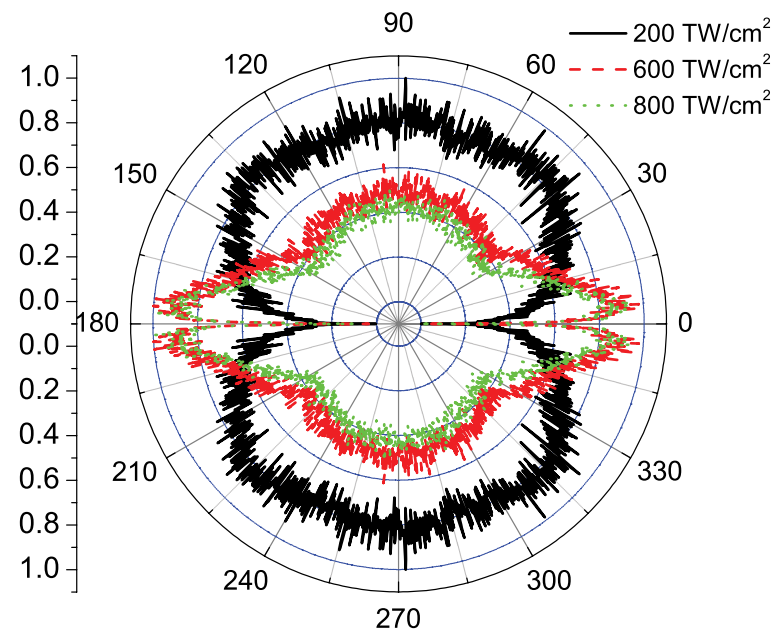

FIG. 7. (Color online) Angular distribution of helium with different intensities in intense laser fields; the wavelength is $2000 \mathrm{~nm}$. 


\section{CONCLUSIONS}

In summary, we have extended the coupled coherent-state method to a nonperturbative $a b$ initio study of strong-field ionization of $6 \mathrm{D}$ helium atoms. The method uses a basis of quantum trajectories guided by frozen Gaussian coherent states, sampled from a Monte Carlo ensemble, as the initial states of the quantum time-dependent Schrödinger equations. The coupled coherent-state trajectories move over averaged potentials, which remove the Coulombic singularities. We observed the low-energy peak structure in helium is in agreement with the experiment [5]. The rescattering event was also demonstrated to be responsible for NSDI by showing a detailed picture of how NSDI happens in terms of the one-particle energy and position distributions of the electrons. In addition, the time evolution of the positions of electrons was also presented, and the results show the rescattering phenomenon in which the recolliding electron returns to the inner electron and then exchanges energy with the inner electron, allowing both to escape. Finally, the electron angular distribution of helium was also investigated. The results show that the angular distribution is very close to the laser axis and the maximum angle becomes smaller when the laser wavelength or intensity is increased.

\section{ACKNOWLEDGMENTS}

This work was partially supported by the Chemical Sciences, Geosciences and Biosciences Division of the Office of Basic Energy Sciences, Office of Sciences, US Department of Energy and by the US National Science Foundation. J.G. would also like to acknowledge the partial support of the Chinese Scholarship Council (CSC). J.G. and X.L. were partially supported by the National Natural Science Foundation of China under Grant No. 10974068.
[1] A. Scrinzi, M. Y. Ivanov, R. Kienberger, and D. M. Villeneuve, J. Phys. B 39, R1 (2006).

[2] M. Drescher, M. Hentschel, R. Kienberger, G. Tempea, Ch. Spielmann, G. A. Reider, P. B. Corkum, and F. Krausz, Science 291, 1923 (2001).

[3] R. Kienberger et al., Science 297, 1144 (2002).

[4] J. Muth-Böhm, A. Becker, and F. H. M. Faisal, Phys. Rev. Lett. 85, 2280 (2000).

[5] C. I. Blaga, F. Catoire, P. Colosimo, G. G. Paulus, H. G. Muller, P. Agostini, and L. F. Dimauro, Nature Phys. 5, 335 (2009).

[6] W. Quan et al. Phys. Rev. Lett. 103, 093001 (2009).

[7] A. Becker and F. H. M. Faisal, Phys. Rev. Lett. 84, 3546 (2000).

[8] L.-B. Fu, J. Liu, and S.-G. Chen, Phys. Rev. A 65, 021406(R) (2002).

[9] J. S. Parker, B. J. S. Doherty, K. J. Meharg, and K. T. Taylor, J. Phys. B 36, L393 (2003).

[10] X. Guan, X. M. Tong, and Shih I Chu, Phys. Rev. A 73, 023403 (2006).

[11] X. M. Tong and Shih I Chu, Chem. Phys. 217, 119 (1997).
[12] J. Liu, D. F. Ye, J. Chen, and X. Liu, Phys. Rev. Lett. 99, 013003 (2007).

[13] R. Panfili, S. L. Haan, and J. H. Eberly, Phys. Rev. Lett. 89, 113001 (2002).

[14] D. V. Shalashilin and M. S. Child, J. Chem. Phys. 113, 10028 (2000).

[15] D. V. Shalashilin and M. S. Child, J. Chem. Phys. 114, 9296 (2001).

[16] D. V. Shalashilin and M. S. Child, J. Chem. Phys. 115, 5367 (2001).

[17] D. V. Shalashilin and M. S. Child, Chem. Phys. 304, 103 (2004).

[18] D. V. Shalashilin and M. S. Child, J. Chem. Phys. 122, 224109 (2005).

[19] D. V. Shalashilin, M. S. Child, and A. Kirrander, Chem. Phys. 347, 257 (2008).

[20] X. S. Liu, Y. Y. Qi, J. F. He, and P. Z. Ding, Commun. Comput. Phys. 2, 1 (2007).

[21] P. B. Corkum, Phys. Rev. Lett. 71, 1994 (1993).

[22] H. R. Reiss, Phys. Rev. A 22, 1786 (1980). 